\title{
Research on the Model of the Radio Wave Propagation in Granary Using Ray-Tracing Algorithm
}

\author{
Chunhua Zhu ${ }^{1, \mathrm{a}}$, Jinkui $\mathrm{YaO}^{2, \mathrm{~b}^{*}}$ \\ ${ }^{1}$ College of Information Science and Engineering, Henan University of Technology, Zhengzhou, \\ 450001, China \\ ${ }^{2}$ College of Information Science and Engineering, Henan University of Technology, Zhengzhou, \\ 450001, China \\ aemail:zhuchunhua@haut.edu.cn, bemail:770428950@qq.com
}

Keywords: ray-tracing; amplitude; delay; angle of arrival; path loss

Abstract. The radio wave propagation model in granary can provide technical support for the design and application of wireless grain situation monitoring and foreign-body detection in granary. The propagation of radio waves in granary with wheat is studied in this paper. Firstly, transmitting and receiving antennas are fixed in grain bulk. Then the parameters of multipath delay and received power at receiver are calculated by using ray-tracing algorithm, and the wave propagation model is established. The results show that only the direct and one-reflection paths of larger amplitude are considered in the wheat grain bulk. Finally, the path losses of simulation at different distances are compared with the theoretical value of the literature, the accuracy of the simulation results is verified and the effect of reflection is reflected.

\section{Introduction}

In recent years, granary monitoring system in temperature and humidity based on wireless sensor network [1], [2] and foreign-body detection sensor system in granary based on GPR(ground penetrating radar) [3], [4] gradually to the development of automation and intelligent, have become a hot topic in the field of grain information processing. The accurate electric wave propagation model and the optimization of physical layer data transmission mode in grain can improve the reliability of the wireless transmission system mentioned above. Nelson S.O [5] researched the feasibility of the pest control in grain storage by using radio waves of different frequency bands in the 1990s, and obtained the attenuation formula of the radio signal transmit in grain. Armstrong Paul [6] studied the feasibility of using the wireless node instead of the traditional scheme of cable in grain monitoring system, the results showed that the signal of $915 \mathrm{MHz}$ in the power consumption of $1 \mathrm{~mW}$ in the grain reliably transmit about 2 meters. Zhang Fenghui [7] of Beijing University of Posts and Telecommunications obtained the energy attenuation value per meter of $780 \mathrm{MHz}$ wireless sensor nodes in wheat grain bulk by using the method of evaluating the energy attenuation of radio waves and statistic of the data packet loss rate. The method and results are of guiding significance for the placement of wireless sensor nodes in wheat grain bulk. The research findings above only consider the large scale fading characteristics at the partial frequency points, the complete wave propagation model is not constructed, and the research method is based on measurement, which is a daunting task and the applicability is bad.

In this paper, the ray-tracing algorithm is used to establish the wave propagation model of the horizontal warehouse environment by calculating the amplitude, delay and angle of arrival of each ray at the receiver under the condition that transmitting and receiving antennas are fixed in the wheat grain bulk.

\section{Modeling process}

\section{Environmental characteristics of granary}

This paper takes the horizontal warehouse as the research object, because the effects of the top can be ignored, the cuboid that long is 4 meters, wide is 3 meters and high is 2.5 meters can be used to simulate the environment. The walls of the four sides and the bottom of the warehouse are 
cement-concrete material, with the dielectric constant 6 and conductivity $0.001 \mathrm{~S} / \mathrm{m}$. The warehouse is full of wheat, high is $2 \mathrm{~m}$. Strictly speaking, the density of different grain layer is different, the closer to the bottom of the warehouse, the greater the density. The temperature and humidity in different storage locations are quite different, the dielectric constant will also change with it, which leads to the extremely complex wave propagation characteristics in granary. In order to simplify the calculation, the grain bulk is treated as a homogeneous medium in this paper. The water content of wheat in granary is about $10 \%$, and corresponding dielectric constant is set to 2.6 , the electrical conductivity is $0.01 \mathrm{~S} / \mathrm{m}$.

Establish a 3 coordinate system with the origin at the bottom of the warehouse, The receiving and transmitting antennas are embedded in the grain bulk. The coordinate of transmitting antenna is set to $(1,1,1)$, the signal frequency used is $780 \mathrm{MHz}$, while the coordinate of receiving antenna is set to $(1$, $3,1)$. Transmitting and receiving antennas are ideal omnidirectional antennas with a gain of 1 .

\section{Propagation path analysis}

In the medium of the grain, radio wave propagation modes between the transmitting antenna and the receiving antenna mainly include direct, reflection, diffraction and scattering, and the main mode is reflection after considering the grain bulk as the homogeneous medium. Taking into account the effect of reflection losses, the paths with four or more reflections have little contribution to the received field strength, so only 1 3 reflections are considered in this paper. All reflections are regarded as specular reflection, i.e., not consider the impact of roughness. According to the principle of images, there are 6 first order images, 30 second order images, 150 third order images for transmitting point, corresponding to the images, there are 6 paths with one-reflection, 30 paths with two-reflections, and 150 paths with three-reflections, add to a direct path, the theoretical value is 187.

\section{Parameters calculation}

The model of ray-tracing propagation [8] is generally expressed as

$$
h(t)=\sum_{n=1}^{N} A_{n} \delta\left(t-\tau_{n}\right) \exp \left(-j \theta_{n}\right) .
$$

where $h(t)$ denotes the channel impulse response; $N$ is the total number of rays received; $\tau_{n}$ is the multipath delay of the $n$ ray; $A_{n}$ and $\theta_{n}$ are the amplitude and phase of the $n$ ray, respectively.

So we can get that in order to get the channel impulse response $h(t), \tau_{n}, A_{n}$ and $\theta_{n}$ of each ray are required to calculate respectively. When the transmitted power is fixed, $A_{n}$ is determined by the received power.

\section{Received power calculation}

Received power can be directly calculated from the received field strength. In a lossy medium, the received field strength is related to the propagation constant.

\section{(1) Field strength}

For the direct ray, the electric field strength is calculated using the following formulas:

$$
E_{D}=E_{0} e^{-\gamma d} \text {. }
$$

where $E_{0}$ is electric field strength at a point of reference, $d$ is propagation path length and $\gamma$ is propagation constant, which is a complex quantity:

$$
\gamma=\alpha+j \beta \text {. }
$$

where $\alpha$ is attenuation constant $(\mathrm{Np} / \mathrm{m}), \beta$ is phase constant $(\mathrm{rad} / \mathrm{m})$. The expressions are given by [9]

$$
\alpha=\omega \sqrt{\frac{\mu \varepsilon}{2}\left[\sqrt{1+\left(\frac{\sigma}{\omega \varepsilon}\right)^{2}}-1\right]} .
$$




$$
\beta=\omega \sqrt{\frac{\mu \varepsilon}{2}\left[\sqrt{1+\left(\frac{\sigma}{\omega \varepsilon}\right)^{2}}+1\right]}
$$

where $\varepsilon$ is the dielectric constant, $\sigma$ is the conductivity, $\mu$ is the magnetic permeability, $\omega=2 \pi f, f$ is the signal frequency.

For low loss medium like grain, loss tangent $T=\frac{\sigma}{\omega \varepsilon} \ll 1$, (4) and (5) can be rewritten as

$$
\begin{aligned}
\alpha & \approx \frac{\sigma}{2} \sqrt{\frac{\mu}{\varepsilon}} . \\
\beta & \approx \omega \sqrt{\mu \varepsilon} .
\end{aligned}
$$

When the incident electromagnetic wave incident upon the interface of the medium of wheat and the wall or air, part of the power is reflected back to the wheat, and the rest is transmitted. The field strength of the reflected wave is usually calculated by the reflection coefficient, which is defined as the ratio of the field strength of the incident wave and reflected wave, mainly related to the dielectric constant, the electromagnetic wave polarization modes and the angle of incidence. Polarization modes of electromagnetic wave include horizontal and vertical polarization. When the electric field of incident wave is perpendicular to the incident plane, it is called TE (Transverse Electric) wave, also known as s or horizontal polarization wave; when the electric field of incident wave is parallel with the incident plane, it is called TM (Transverse Magnetic) wave, also known as p or vertical polarization wave. In different polarization modes, the reflection coefficients have different expression. The expressions of S-P component of Fresnel reflection coefficient [10] are

$$
\begin{aligned}
R_{s} & =\frac{n_{1} \cos \theta_{i}-n_{2} \cos \theta_{t}}{n_{1} \cos \theta_{i}+n_{2} \cos \theta_{t}} . \\
R_{p} & =\frac{n_{2} \cos \theta_{i}-n_{1} \cos \theta_{t}}{n_{2} \cos \theta_{i}+n_{1} \cos \theta_{t}} .
\end{aligned}
$$

where $n_{1}$ and $n_{2}$ are the refractive index of the incident and the refractive medium respectively; $\theta_{i}$ and $\theta_{t}$ are the angle of incidence and refraction respectively.

Using refraction law $n_{1} \sin \theta_{i}=n_{2} \sin \theta_{t}$ to eliminate the angle of refraction $\theta_{t}$, the expression above can be changed to

$$
\begin{aligned}
& R_{s}=\frac{\cos \theta_{i}-\sqrt{\varepsilon_{R}-\sin ^{2} \theta_{i}}}{\cos \theta_{i}+\sqrt{\varepsilon_{R}-\sin ^{2} \theta_{i}}} . \\
& R_{p}=\frac{\varepsilon_{R} \cos \theta_{i}-\sqrt{\varepsilon_{R}-\sin ^{2} \theta_{i}}}{\varepsilon_{R} \cos \theta_{i}+\sqrt{\varepsilon_{R}-\sin ^{2} \theta_{i}}} .
\end{aligned}
$$

where $\varepsilon_{R}=\varepsilon_{r}-j 60 \sigma \lambda, \varepsilon_{r}$ is the ratio of the dielectric constant of the incident and refractive medium, $\sigma$ is the special conductivity of the reflecting surface.

For the plane harmonic of the general polarization, it can be decomposed into vertical and horizontal polarized waves, the field strength [9] of the reflected rays can be written as

$$
E_{R}=\left[\left(E_{D} * \vec{e}_{\perp}\right) R_{s}\right] \vec{e}_{\perp}+\left[\left(E_{D} * \vec{e}_{/ /}\right) R_{p}\right] \vec{e}_{/ /} .
$$

where $\vec{e}_{\perp}$ and $\vec{e}_{/ /}$are the reflection unit vector of horizontal and vertical polarization respectively.

(2) Received power

Received power can be calculated as[12].

$$
P_{r}=P D \cdot A_{e} \text {. }
$$

where $P D$ is the radiation power density of the transmitting antenna, $P D=E^{2} / 2 \eta$; 
$\eta$ is the inherent impedance of the medium, $\eta=\sqrt{\mu / \varepsilon}$;

$A_{e}$ is the effective area of the receiving antenna, $A_{e}=\frac{G_{R} \lambda^{2}}{4 \pi}$;

$G_{R}$ is the gain of receiving antenna.

So received power is given by

$$
P_{r}=\frac{E^{2} G_{R} \lambda^{2}}{8 \pi \eta} \text {. }
$$

\section{Delay and arrival angle calculation}

The value of delay is the difference between the time of propagation of the reflected path and the direct path. The angle of arrival is defined as the angle formed by the paths that reach the receiving point and the projection of the direct path in the $\mathrm{Z}$ plane.

In the medium of wheat, propagation velocity of electromagnetic wave is no longer the speed of light, but related to the relative permittivity of the medium $\varepsilon^{\prime}$.

$$
v=\frac{1}{\sqrt{\mu \varepsilon}}=\frac{c}{\sqrt{\varepsilon^{\prime}}} .
$$

The total length of each path with arbitrary number of reflections is the distance between the receiving point and the corresponding highest order image, which can calculate the propagation time. Then the multipath delay can be obtained by subtracting the propagation time of direct path.

\section{Results and analysis}

Based on the parameters calculation method of the 2.3 section, the amplitude values of the received rays are shown in Fig. 1. It can be seen that the number of effective rays are 47, and the amplitude of direct path is largest. Relative to the direct and one-reflection multipath components, the amplitude of rays with two and three reflections are extremely small, the order of magnitude is $10^{-6} \sim 10^{-9}$, which have little contribution to the received field. Therefore, only the direct path and paths with one-reflection are considered in the practical application.

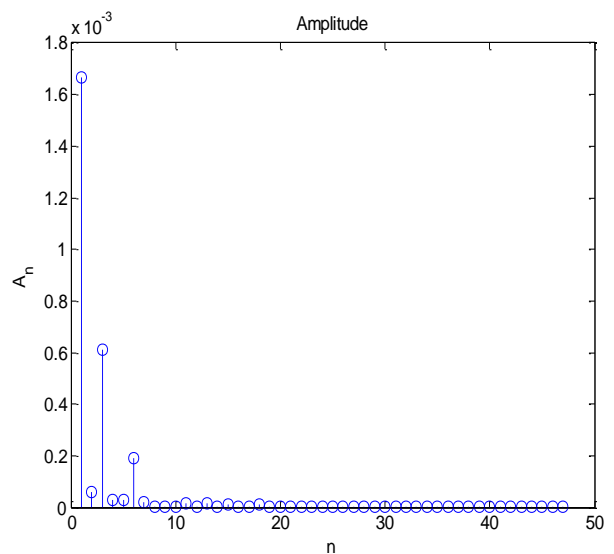

Fig. 1 The amplitude of rays

The additional delay and the angle of arrival of the direct and one-reflection paths are shown in Fig. 2 and Fig. 3. For the angle of arrival, in order to distinguish the propagation path of different direction, the angle of arrival of the rays from the top of the antenna to the receiving point are set to be positive, on the contrary, be negative. 


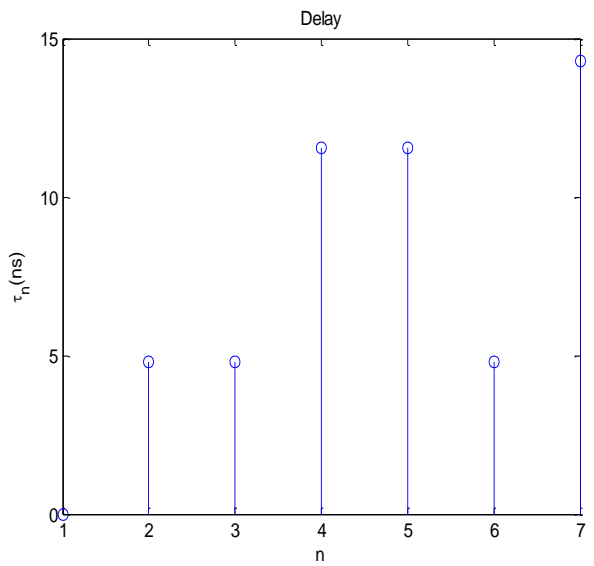

Fig. 2 Multipath delay

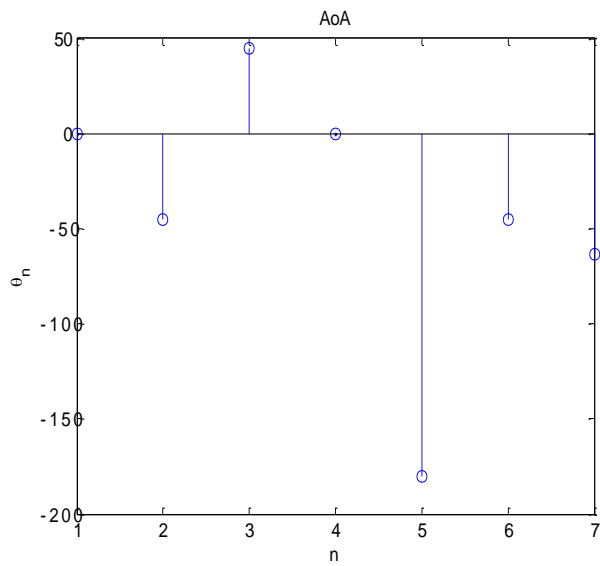

Fig.3 Angle of arrival

In literature [5], the attenuation formula of radio signal in grain is presented:

$$
\alpha=\frac{8.686 \pi \varepsilon^{\prime \prime}}{\lambda_{0} \sqrt{\varepsilon^{\prime}}} \mathrm{dB} / \mathrm{m} \text {. }
$$

where $\alpha$ represents the signal attenuation, $\varepsilon^{\prime}$ and $\varepsilon^{\prime \prime}$ are the real and imaginary parts of dielectric constant of grain, $\varepsilon^{\prime \prime}=\sigma / \omega \varepsilon_{0}$.

Since the power dissipated is proportional to $E^{2}$, the path loss $P_{L}$ can be given by

$$
P_{L}=10 \log _{10}\left(\frac{P_{t}}{P_{r}}\right)=20 \log _{10}\left(\frac{\left|E_{0}\right|}{\left|E_{\text {tot }}\right|}\right) \text {. }
$$

where $E_{t o t}$ is the total received field which is vector addition of field strength of all components.

Change the position coordinates of the receiving antenna from $(1,1.1,1)$ to $(1,2.5,1)$ at $0.1 \mathrm{~m}$ intervals. The 15 total field strength values can be obtained. The path loss of the 15 positions are calculated, and then compare them with (16), the result is shown in Fig. 4. It can be seen that the simulative value is almost identical with the theoretical value within $1.5 \mathrm{~m}$ and less than the theoretical value in the distance between $1.5 \mathrm{~m}$ to $2 \mathrm{~m}$. The reason for the result of incomplete coincidence is that the theoretical value is based on the premise that the grain bulk is regarded as an infinite uniform space, only direct path is considered, while the simulation environment of this paper is the space of limited shape, and considering both direct and reflected paths. Thus the fluctuation of the simulation curve with respect to the theoretical curve is caused by the reflection of the walls of the warehouse, and when the distance is more than $1.5 \mathrm{~m}$, the effect of reflection on the results is more obvious.

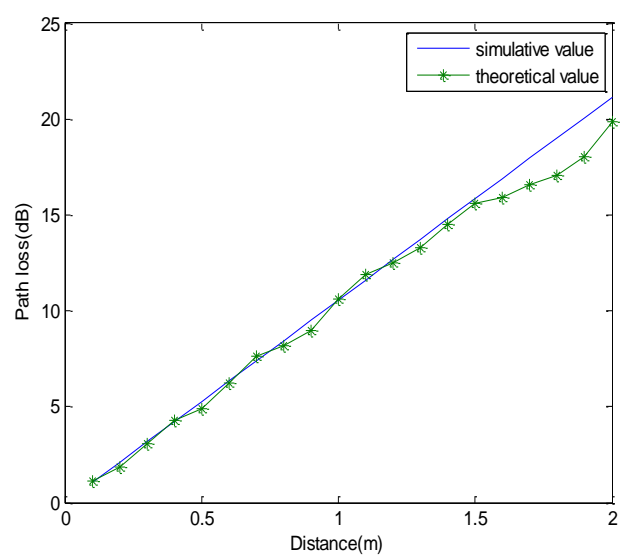

Fig.4 Comparison of simulative and theoretical path loss 


\section{Conclusions}

In this paper, the radio wave propagation characteristics in the horizontal warehouse with wheat are studied by using ray-tracing algorithm, the calculation method of the field strength, received power, delay and arrival angle of the multipath components in the receiving point are given in detail. Through the amplitude of multipath components, we can see that the paths with two-reflections and three-reflections have a very small contribution to the receiving field, so that only the direct path and paths with one-reflection are considered, which shows the high loss of electromagnetic wave in the grain bulk. At the same time, by comparing the simulative path loss with the theoretical value in the literature, the accuracy of the simulation is verified and the effect of reflection is reflected. Compared with the attenuation formula in the literature, the simulation results of this paper have more reference value. It is worth noting that the model of wave propagation in the medium of wheat in this paper is under the assumption that the granary environment is specific and electromagnetic properties are uniform, the characteristics of radio wave propagation in the medium of wheat in different granary need to be further studied.

\section{Acknowledgement}

This work was supported by National food industry commonweal special scientific research projects (201413001) and Key Science and Technique Project of He'nan Educational Committee (14A510014) and the National Natural Science Foundation of China under Grant (61601170).

\section{References}

[1] Huang Zhihua. The design and research of intelligent grain situation monitoring system in grain depot. University Of Anhui, 2015.

[2] Yi Cuiping. Research on granary monitoring system in temperature and humidity based on Wireless Sensor Network. Changchun University of Science and Technology, 2012.

[3] Lian Feiyu, Fu Maixia. Research on foreign-body detection in granary using ground penetrating radar technique. Journal of Henan University of Technology (Natural Science Edition), 2008,29(4),50 55.

[4] Liu Hua, Chen Xianxiang. GprMax simulation of microwave detection sensor system of foreign matter in grain depot. Journal of Henan University of Technology (Natural Science Edition), 2008,29(6),60 64.

[5] Nelson S O. Review and assessment of radio frequency and microwave energy for stored grain insect control. Transactions of the ASAE, 1996, 39(4): 1475-1484.

[6] Armstrong Paul. Wireless data transmission of networked sensors in grain storage[C]//The 2003 ASAE Annual International Meeting. July27- 30 2003, Riviera Hotel and Convention Center Las Vegas, Nevada, USA: ASABE2003: 1-12.

[7] Zhang Fenghui, Zhou Huiling, Zhang Fengying, Zhou Xiaoguang. Reliable communication of wireless sensor nodes in grain bulk. Journal of Henan University of Technology (Natural Science Edition), 2010,31(4): 54 57.

[8] R.A Valenzuela. A Ray Tracing Approach to Predicting Indoor Wireless Transmission. Proceeding 43rd IEEE vehicular Technology Conference, 1993:214 218.

[9] Frank Gross. Smart antennas for wireless communications. Beijing: Publishing House of Electronics Industry, 2009. 
[10]Wei Lihong. The relationship between the $\mathrm{S}$ component and $\mathrm{P}$ component of the Fresnel reflection coefficient and the incident angle. Songliao Journal(Natural Science Edition),1988, 3:74 75.

[11]Dikshitulu K. Kalluri. Electromagnetic waves. Beijing: China Machine Press, 2014.

[12]Larry Burgess. Measuring radiated power and field strength from UHF ISM transmitters. Electronic technology application. 2007,(11), 89 90. 